\title{
Enhanced thermo-spin effects in iron-oxide/metal multilayers
}

\author{
R Ramos ${ }^{1}$, I. Lucas ${ }^{2,3,4}$, P. A. Algarabel ${ }^{4,5}$, L. Morellón ${ }^{2,3,4}$, K. Uchida ${ }^{6,7,8}$, E. \\ Saitoh $^{1,8,9,10}$ and M. R. Ibarra ${ }^{2,3,4,11}$
}

1. Advanced Institute for Materials Research, Tohoku University, Sendai 980-8577, Japan

2. Fundación Instituto de Nanociencia de Aragón, E-50018 Zaragoza, Spain

3. Instituto de Nanociencia de Aragón, Universidad de Zaragoza, E-50018 Zaragoza, Spain

4. Departamento de Física de la Materia Condensada, Universidad de Zaragoza, E-50009 Zaragoza, Spain

5. Instituto de Ciencia de Materiales de Aragón, Universidad de Zaragoza and Consejo Superior de Investigaciones Científicas, 50009 Zaragoza, Spain

6. National Institute for Materials Science, Tsukuba 305-0047, Japan

7. PRESTO, Japan Science and Technology Agency, Saitama 332-0012, Japan

8. Center for Spintronics Research Network, Tohoku University, Sendai 980-8577

9. Institute for Materials Research, Tohoku University, Sendai 980-8577, JapanAdvanced Science

10. Advanced Science Research Center, Japan Atomic Energy Agency, Tokai 319-1195, Japan

11. Laboratorio de Microscopías Avanzadas, Universidad de Zaragoza, E-50018 Zaragoza, Spain

\begin{abstract}
Since the discovery of the spin Seebeck effect (SSE), much attention has been devoted to the study of the interaction between heat, spin, and charge in magnetic systems. The SSE refers to the generation of a spin current upon the application of a thermal gradient and detected by means of the inverse spin Hall effect. Conversely, the spin Peltier effect (SPE) refers to the generation of a heat current as a result of a spin current induced by the spin Hall effect. Here we report a strong enhancement of both the SSE and $\mathrm{SPE}$ in $\mathrm{Fe}_{3} \mathrm{O}_{4} / \mathrm{Pt}$ multilayered thin films at room temperature as a result of an increased thermo-spin conversion efficiency in the multilayers. These results open the possibility to design thin film heterostructures that may boost the application of thermal spin currents in spintronics.
\end{abstract}




\section{Introduction}

Thermoelectric effects have been known since the discovery of the Seebeck effect (SE) [1], which is manifested as the generation of an electrical field $(\mathbf{E})$ in a metal or semiconductor due to a thermal gradient $(\nabla T)$, resulting in a conversion from heat to electricity. Large expectation of the implementation of this principle for clean energy applications has been limited by the scarce improvement of the efficiency of the thermoelectric conversion, which is described by the figure of merit $Z T=\frac{S^{2} \sigma}{\kappa} T$, where $T$ is the system temperature, $S=\frac{\vec{E}}{\nabla T}$ is the Seebeck coefficient, $\sigma$ is the electrical conductivity, $\kappa$ the thermal conductivity. In principle, the thermoelectric efficiency can be enhanced by simultaneously increasing $\sigma$ and decreasing $\kappa$ of a material. However, when $\kappa$ is dominated by the electronic contribution $\kappa_{e}$, it is difficult to change these quantities independently in a material due to the Wideman-Franz law $\kappa_{e}=L \sigma T$, where $L$ is the Lorenz number. Similar argument applies to the Peltier effect, the Onsager reciprocal of the SE, i.e. the conversion of an applied electrical field into a heat flow and described by: $\Pi=S T$, where $\Pi$ is the Peltier coefficient. Several other approaches to improve the thermoelectric conversion performance are currently being investigated [2]. Another posible route to explore is the study of the interaction between the heat, charge, and spin degrees of freedom in magnetic materials $[3,4]$.

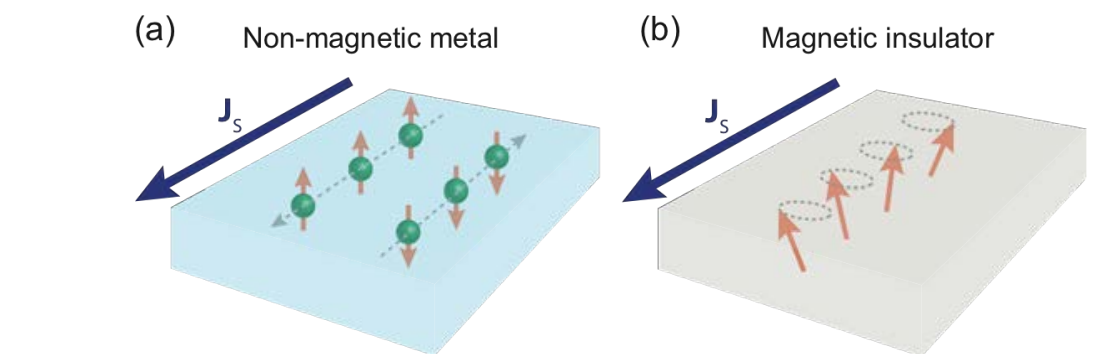

Figure 1. Schematic diagram depicting (a) conduction-electron and (b) magnon spin currents

Recently a new paradigm has gained increased attention: the generation and manipulation of spin currents $\left(J_{S}\right)[5,6]$. This concept relies in the existence of a flow of spin angular momentum without net flow of charge. Spin currents in metals or semiconductors are originated by a net flow of the spin angular momentum of the electrons and called electron spin currents. The existence of a spin current does not necessarily imply the existence of free electrons, as in the former case, but it is also possible to have spin currents in insulating ferromagnets with localized magnetic moments [7, 8]. In this case, the magnons provide the excitation mechanism to carry spin angular momentum in the system and generate spin currents that are called magnon spin currents. (see a schematic view of both type of spin currents in figure 1).

Recent advances in this field were made possible thanks to the possibility to generate and detect these spin currents, $J_{\mathrm{S}}$. Pioneer experiments of spin current generation were done using radiofrequency excitation in ferromagnetic materials, a phenomenon called spin-pumping [9, 10]. Subsequently, the discovery of the spin Seebeck effect (SSE) revealed that a temperature gradient in a ferromagnet (either metallic or insulator) can originate thermal spin currents [7, 11, 12, 13]. Others mechanisms, such as excitation by acoustic waves have also been demonstrated to induce spin currents in ferromagnets [14, $15,16]$. The detection of the $J_{\mathrm{S}}$ is determined by the electrical field that can induce in a metal with large 
spin orbit coupling, such as Pt, adjacent to the ferromagnet, and called the inverse spin Hall effect (ISHE) $[9,17,18,19,20]$. More recently, the Onsager-reciprocal of the SSE has also been experimentally demonstrated, this is the spin Peltier effect (SPE) [21, 22, 23, 24, 25], which consists on the generation of a heat current as a result of a spin current injection into the ferromagnet.

This review aims to show the relevance of the thermo-spin effect in magnetic thin film multilayers of iron oxide and Pt. Here, we report the thermo-spin effect in magnetite (low electrical conductivity) and maghemite (insulator)/Pt bilayers. These are binary iron oxides crystallizing in a spinel-type structure having chemical formulae $\mathrm{Fe}_{3} \mathrm{O}_{4}$ and $\gamma-\mathrm{Fe}_{2} \mathrm{O}_{3}$, respectively [26, 27, 28]. The presence of additional $\mathrm{Fe}^{2+}$ is responsible for the moderate electrical conductivity in $\mathrm{Fe}_{3} \mathrm{O}_{4}$ [28] and magneto-resistive properties $[29,30,31]$. The study of the spin current generation in these oxide systems is interesting due to the high abundance of its constituent elements ( $\mathrm{Fe}$ and $\mathrm{O}$ ), the possibility of fabrication of hetero-epitaxial structures [32] and their higher Curie temperatures $\left(848 \mathrm{~K}\right.$ and $900 \mathrm{~K}$ for $\mathrm{Fe}_{3} \mathrm{O}_{4}$ and $\gamma-\mathrm{Fe}_{2} \mathrm{O}_{3}$, respectively), which could be of potential interest for thermoelectric conversion applications.

We found that the SSE is driven by the thermally generated spin currents in both iron oxides. Moreover, in $\left[\mathrm{Fe}_{3} \mathrm{O}_{4} / \mathrm{Pt}\right]_{\mathrm{n}}$ multilayers the SSE and SPE are strongly enhanced, which can be understood as a result of a spin current enhancement within the multilayer thickness. We also compare the thermoelectric power response of spin-Hall thermopile devices and multilayers in which we showed that the design based in the use of multilayer can achieve the largest power factor found using SSE. Finally, we briefly review the SSE in a variety of other magnetic oxides.

\section{Basic mechanism and measurement procedure of thermo-spin effect:}

\subsection{Spin Seebeck effect (SSE)}

Conventionally, the spin Seebeck effect consists on the generation of a spin current in a bilayer system comprising a ferro-, ferri-magnetic material (FM) and a normal metal (NM) via the magnetization dynamics induced by the application of a thermal gradient. The spin current injected in the NM layer is electrically detected (SSE voltage) by the inverse spin Hall effect (ISHE) as a result of the spin-orbit interaction in the NM layer (see figure 1(a)) $[9,17,18,19,20]$. To have a better detection efficiency, the NM is usually a heavy metal, mainly Pt $[33,34]$. The ISHE-driven electric field $\left(\mathbf{E}_{\text {ISHE }}\right)$ is given by the following expression:

$$
\mathbf{E}_{\mathrm{ISHE}}=\theta_{\mathrm{SH}} \rho\left(\mathbf{J}_{\mathrm{S}} \times \boldsymbol{\sigma}\right)
$$

where $\theta_{\mathrm{SH}}$ and $\rho$ denote the spin Hall angle and electric resistivity of the NM layer. $\mathbf{J}_{\mathrm{S}}$ and $\boldsymbol{\sigma}$ are the spatial direction of the spin current injected into NM across the FM/NM interface (parallel to $\nabla T$ ) and the spin polarization (parallel to the magnetization of the FM), respectively. 


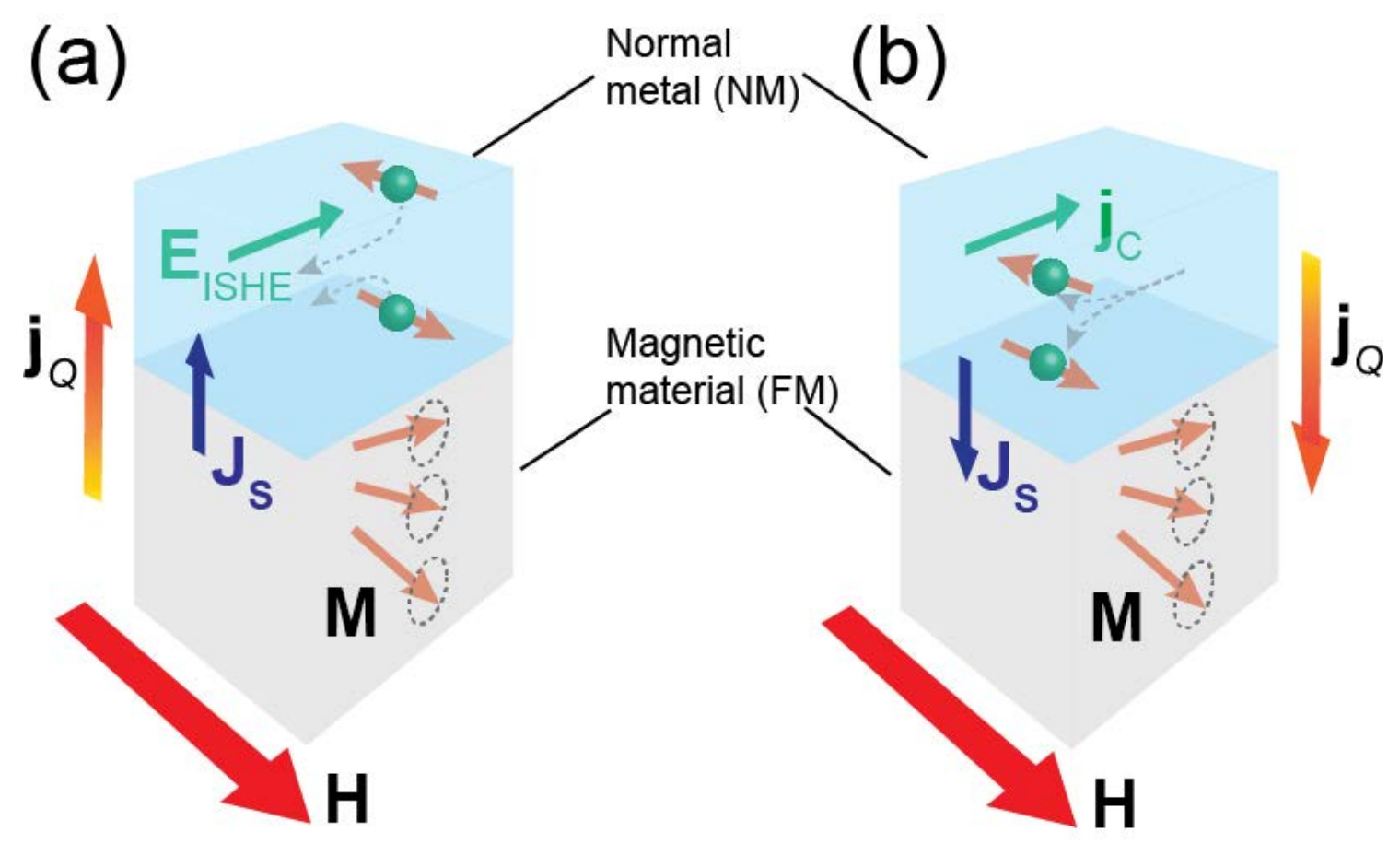

Figure 2. Schematic diagram representing the basic (a) spin Seebeck effect (SSE) and (b) spin Peltier effect (SPE) mechanism in a FM/NM bilayer with the inverse spin Hall effect (ISHE) and spin Hall effect (SHE) of the NM layer, respectively. In the SSE a thermal excitation drives a spin current while in the SPE an injected spin current induces a heat current at the FM/NM interface. The symbols: $\mathbf{E}_{\mathrm{ISHE}}, \mathbf{H}, \mathbf{M}, \mathbf{j}_{\mathrm{O}}$, and $\mathbf{J}_{\mathrm{S}}$ denote the ISHE-induced electric field, magnetic field, magnetization, spatial direction of heat and spin currents, respectively.

The SSE was initially observed in a $\mathrm{Ni}_{81} \mathrm{Fe}_{19}$ film [11], and it was theoretically formulated in terms of conduction electrons. However, the observation of the SSE in magnetic insulators pointed to its magnonic origin, due to the thermal excitation of localized spins in the ferromagnet. The theoretical framework was then developed [35, 36, 37] to explain the SSE by the thermal non-equilibrium between magnons in the FM and electrons in the NM, and the magnitude of the generated spin current was explained in terms of the difference of the effective temperatures between the magnon and electron systems. This model only considered the SSE as a result of an effective temperature difference at the interface, however experimental observations, such as the dependence of the SSE voltage on the thickness of the ferromagnet [38, 39] and its magnetic field induced suppression [39, 40, 41, 42], called for a refinement of the model. Then, Rezende et al. [43, 44] and Zhang et al. [45, 46] independently formulated the SSE by the thermally induced bulk magnon spin currents within the ferromagnet, not just at the interface. Basically, the applied heat drives the magnon system out of thermal equilibrium, thus generating a magnon accumulation. This results in the generation of a magnon spin current which is dependent on both; the applied temperature gradient and the induced magnon accumulation due to non-equilibrium magnons. Using their model several aspects of the SSE can be explained, such as the dependence on the FM thickness [38, 39], the temperature dependence [39, 43], the suppression of the SSE at high magnetic fields [39, 40, 41, 42], the multilayer SSE [47, 48, 49] (which is described below) and the observation of the SSE in antiferromagnetic materials [50, 51, 52, 53]. Other models have also been developed to explain different aspects of the SSE [54-68].

The SSE can be measured in two different experimental arrangements, these are the transverse and longitudinal SSEs, their names originate from the relative direction between the spin current and the applied temperature gradient, being perpendicular/parallel for the transverse/longitudinal SSE (see figure 3(a)/3(b)). The first experimental observation of the SSE in $\mathrm{Ni}_{81} \mathrm{Fe}_{19}$ was performed employing 
the transverse SSE geometry, in this configuration the thermal excitation of the magnetization induces a spin current injection across the FM/NM interface, and it is detected by the ISHE in an attached Pt wire. In principle, the transverse SSE can be used to detect the SSE in magnetic materials independently of their electronic transport properties: metals, semiconductors, insulators [7, 11, 69, 70, 71]. However, this configuration is very sensitive to the thermal design of the measurement system, which can result in a voltage locally-driven by the longitudinal SSE and/or spurious voltages due to conventional spin dependent thermoelectric effects $[72,73,74,75]$.

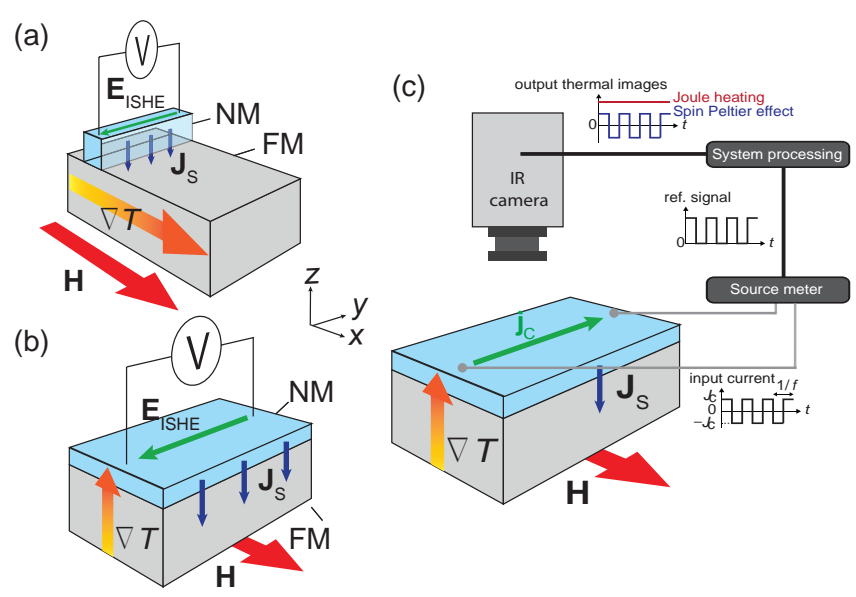

Figure 3. Schematic of the experimental configuration for the measurement of the (a) transverse, (b) longitudinal SSE and (c) thermal imaging of the SPE effect

Due to the above-mentioned complications with the transverse SSE, in this article we will focus on measurements in the longitudinal SSE (see figure 3(b)), which we will simply refer to as the SSE hereafter. Due to its simplicity, this configuration is the most commonly employed to experimentally investigate the SSE. Other possible experimental method is the non-local SSE, which is described elsewhere $[76,77,78]$. In the longitudinal SSE, the injected spin current is parallel to the thermal gradient, applied in the direction normal to the NM/FM interface, along $z$ in figure 3(b), the magnetic field is directed in the $x$ direction and the electric voltage detected in the $y$ direction. This experimental configuration is similar to the one employed to measure the anomalous Nernst effect (ANE) in electrically conductive ferromagnets [79, 80, 81, 82, 83, 84, 85]. Therefore, this configuration should be ideally employed to measure the SSE of electrically insulating materials, since in conductive materials the SSE and ANE are entangled. However, we have previously shown that in ferromagnetic materials with poor electrical conductivity, the SSE dominates the spin-driven thermoelectric response due to the suppression of the ANE-driven voltage of the FM by the larger electrical conductivity of the NM layer, as described in section 3.2.1 [86].

\subsection{Spin Peltier effect (SPE)}

The spin Peltier effect (SPE) is the Onsager-reciprocal of the SSE, it consists on the generation of a heat current in response to a spin current injection across a FM/NM interface (parallel to the surface normal n) $[21-25,87,88,89]$. The spin current is normally generated by means of the spin Hall effect (SHE) of the NM layer, such as Pt and W $[33,34]$, where a charge current $\left(\mathbf{j}_{\mathrm{C}}\right)$ induces a spin accumulation: $\mu_{\mathrm{S}}(\|$ $\sigma) \propto \theta_{\mathrm{SH}} \mathbf{j}_{\mathrm{C}} \times \mathbf{n}$ and a spin angular momentum flow (or spin current $\mathbf{j}_{\mathrm{s}}$ ) at the FM/NM interface via the interfacial exchange coupling (see figure 1(b)). The injected spin current generates a magnon excitation in the ferrimagnet, increasing or decreasing the number of magnons depending on the relative orientation 
between $\boldsymbol{\sigma}$ and $\mathbf{M}$ (parallel or antiparallel), this induces a temperature difference due to energy transfer by the spin current injection at the FM/NM interface, which can be understood by the following relation:

$$
\Delta T_{\mathrm{SPE}} \propto \boldsymbol{\mu}_{\mathrm{S}} \cdot \mathbf{m} \propto\left(\mathbf{j}_{\mathrm{C}} \times \mathbf{n}\right) \cdot \mathbf{m},
$$

For a more detailed theoretical description of the SPE, the reader is referred to the recently developed theoretical models $[87,88,89]$.

The SPE-driven temperature difference in FM/NM junctions has been detected by means of two different experimental methods: thermocouple sensors [21, 25] and lock-in thermography [22, 23, 24]. In this paper, we review measurements performed using the lock-in thermography method [90, 91]. Here, the infrared thermal radiation emitted from the sample surface is measured by an infrared (IR) camera while a rectangular wave charge current with amplitude $J_{\mathrm{C}}$, frequency $f$ is applied to the Pt layer of the samples in the $y$ direction, and an in-plane magnetic field $\mathbf{H}$ with magnitude $H$ is applied in the $x$ direction (see figure 3(c)). The obtained thermal images are transformed into the lock-in amplitude $A$ $(A>0)$ and phase $\phi(0<\phi<360)$ images by Fourier analysis. By extracting the first harmonic response of the thermal images the contributions from the SPE (time varying response) ( $\propto J_{\mathrm{C}}$ ) and Joule-heating (constant in time) $\left(\propto J_{\mathrm{C}}{ }^{2}\right)$ can be separated. The detected IR intensity is converted into temperature information following the calibration method detailed in the supplementary information of [22].

\section{Spin Seebeck effect in Fe-based binary spinel oxides:}

\section{$3.1 \gamma-\mathrm{Fe}_{2} \mathrm{O}_{3} /$ Pt system}

We have investigated the SSE of $\gamma-\mathrm{Fe}_{2} \mathrm{O}_{3}$, an electrical insulator with a band gap of $\sim 2.0 \mathrm{eV}$ [26]. Both $\gamma-\mathrm{Fe}_{2} \mathrm{O}_{3}$ and $\mathrm{Fe}_{3} \mathrm{O}_{4}$ have similar crystal structures, with the difference that only $\mathrm{Fe}^{3+}$ cations are present in $\gamma-\mathrm{Fe}_{2} \mathrm{O}_{3}$ and charge neutrality is achieved by the presence of cation vacancies in the octahedral sites [26]. $\gamma-\mathrm{Fe}_{2} \mathrm{O}_{3}$ is a well-known ferrimagnetic material with a high Curie temperature $\sim 900 \mathrm{~K}$, which has been used in magnetic recording technologies [27].

The films shown here were obtained by in-situ oxygen annealing of $\mathrm{Fe}_{3} \mathrm{O}_{4}$ films, previously deposited by pulsed laser deposition (PLD), for $2.5 \mathrm{~h}$ under different oxygen partial pressures. Figures 4(a) and 4(b) show the results of the X-ray diffraction and temperature dependence of the magnetization, respectively. After oxygen annealing, the (004) peak reflection shifts to a higher angle with a value in reasonable agreement to that of $\gamma-\mathrm{Fe}_{2} \mathrm{O}_{3}$. Moreover, the Verwey transition [28] observed in magnetization measurements for $\mathrm{Fe}_{3} \mathrm{O}_{4}$ film (figure 4(b)) disappears upon oxygen annealing, as expected. For more details about sample fabrication procedure and characterization see Ref. [92].

The results of the SSE measurements are shown in figure 5(c), we can see that the magnitude of the measured voltage is quite similar for all the samples, with a slight increase for the sample annealed under higher oxygen partial pressure. This result suggests that both oxide films possess similar spin current generation properties $[92,93]$, therefore in the rest of this review we will focus on the results obtained in bilayers and multilayers based on $\mathrm{Fe}_{3} \mathrm{O}_{4}$ due to the simplicity of sample preparation. 

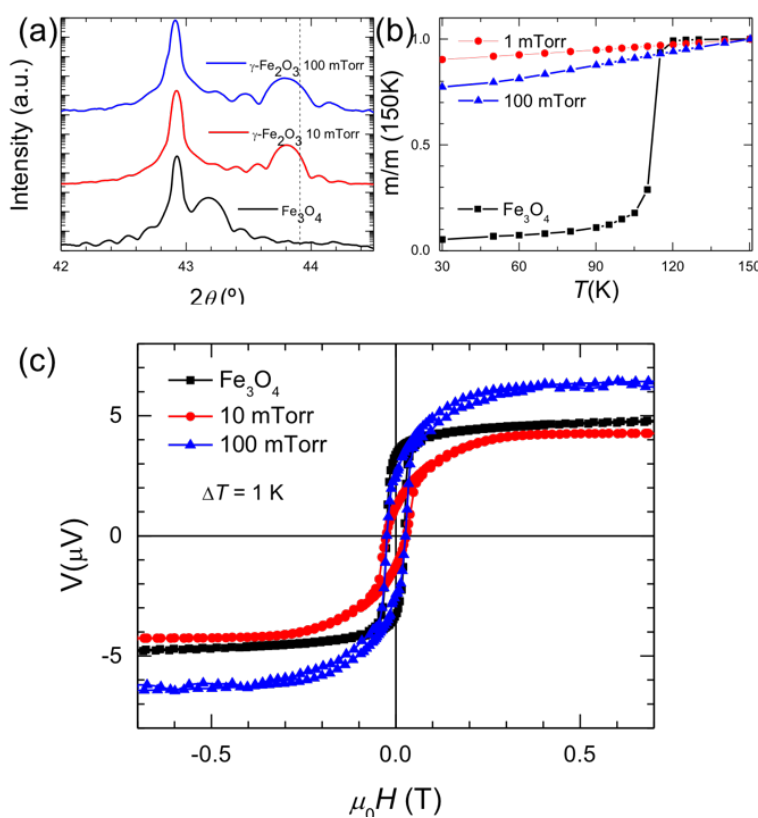

Figure 4. (a) X-ray diffraction patterns around the $\mathrm{MgO}(002)$ reflection showing the (004) reflections for $\mathrm{Fe}_{3} \mathrm{O}_{4}$ and $\gamma$ - $\mathrm{Fe}_{2} \mathrm{O}_{3}$ under different oxygen annealing pressures. The dashed line shows the expected (004) reflection of $\gamma-\mathrm{Fe}_{2} \mathrm{O}_{3}$ after considering a constant volume unit cell. (b) Temperature dependence of the magnetization measured under a constant external magnetic field $\mu_{0} \mathrm{H}=0.05 \mathrm{~T}$ (characteristic Verwey transition at $T \sim 110 \mathrm{~K}$ is present for $\mathrm{Fe}_{3} \mathrm{O}_{4}$ film [28]). (c) SSE-induced voltage for $\mathrm{Fe} 3 \mathrm{O} 4$ and $\gamma-\mathrm{Fe}_{2} \mathrm{O}_{3}$ annealed under different oxygen partial pressures. The thickness of the iron oxides is $60 \mathrm{~nm}$ and the Pt thickness is $7 \mathrm{~nm}$. Adapted from Jiménez-Cavero et al. [92].

\section{$3.2 \mathrm{Fe}_{3} \mathrm{O}_{4} / \mathrm{Pt}$ system}

\subsubsection{Bilayers}

As previously discussed, the SSE in the longitudinal configuration and the ANE share the same experimental geometry. The ANE induced electric field $\left(\mathbf{E}_{\mathrm{ANE}}\right)$ is given by:

$$
\mathbf{E}_{\mathrm{ANE}}=Q_{\mathrm{ANE}} \mu_{0}(\nabla T \times \mathbf{M})
$$

were $Q_{\mathrm{ANE}}, \mu_{0}, \nabla T$, and $\mathbf{M}$ are the ANE coefficient, vacuum permeability, applied thermal gradient and magnetization of the sample, respectively. We can see that this expression has a clear resemblance to that of the ISHE-induced electric field in an in-plane magnetized (IM) configuration (see equation (1)). Therefore, when measuring the SSE in non-insulating ferromagnets, the contributions from ANE and SSE can be entangled [94, 95, 96]. However, we have previously shown that, when the electrical conductivity of the ferromagnet is comparatively lower than that of the normal metal, the SSE dominates the transverse thermoelectric voltage response, as a consequence of the suppression of the ANE voltage of the ferromagnet by the larger electrical conductivity of the NM layer [86]. Since $\mathrm{Fe}_{3} \mathrm{O}_{4}$ has a moderate electrical conductivity due to electron hopping between the octahedral-coordinated $\mathrm{Fe}$ cations due to their mixed electronic valences (equal number of $\mathrm{Fe}^{2+}$ and $\mathrm{Fe}^{3+}$ ) [28], an ANE-driven transverse thermoelectric voltage response is present [84]. However, due to the electrical resistivity of $\mathrm{Fe}_{3} \mathrm{O}_{4}\left(\rho_{\mathrm{Fe} 304}=6.94 \times 10^{-5} \Omega \mathrm{m}\right)$ being about two-orders of magnitude larger than that of $\mathrm{Pt}\left(\rho_{\mathrm{Pt}}=1.39 \times 10^{-}\right.$

${ }^{7} \Omega \mathrm{m}$ ), in the $\mathrm{Fe}_{3} \mathrm{O}_{4} / \mathrm{Pt}$ junction system the contribution from the ANE of $\mathrm{Fe}_{3} \mathrm{O}_{4}$ to the transverse thermoelectric voltage is strongly suppressed. We have measured the ANE and SSE in $\mathrm{Fe}_{3} \mathrm{O}_{4}$ layer and $\mathrm{Fe}_{3} \mathrm{O}_{4} / \mathrm{Pt}$ bilayers (see figure 5) with thicknesses $t_{\mathrm{Fe} 304}=34 \mathrm{~nm}$ and $t_{\mathrm{Pt}}=17 \mathrm{~nm}$, and found that the ANE 
is strongly suppressed with the SSE giving the dominant contribution to the transverse thermoelectric voltage in the $\mathrm{Fe}_{3} \mathrm{O}_{4} / \mathrm{Pt}$ system [85]. More details about sample preparation can be found elsewhere [47].

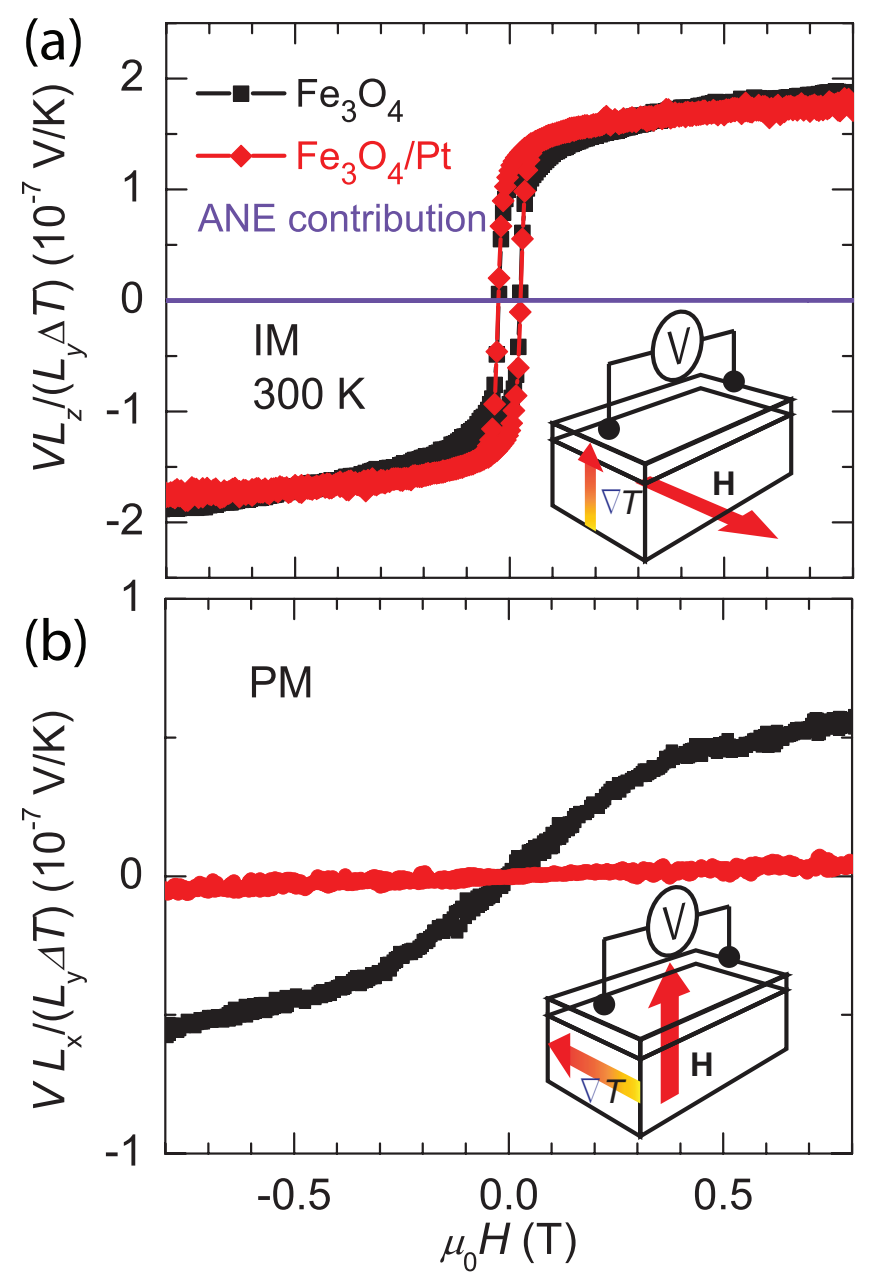

Figure 5. Transverse thermoelectric voltage measured at $300 \mathrm{~K}$ in the (a) in-plane magnetized (IM) and (b) perpendicular magnetized (PM) configuration on a $\mathrm{MgO}(001) / / \mathrm{Fe}_{3} \mathrm{O}_{4}(34)$ and $\mathrm{MgO}(001) / / \mathrm{Fe}_{3} \mathrm{O}_{4}(34) / \mathrm{Pt}(17) S S E$ heteroepitaxial thin films (thickness in $\mathrm{nm}$ ). The insets show the experimental geometry configuration in each case. The purple line in (a) indicates the estimated contribution from the ANE of the $\mathrm{Fe}_{3} \mathrm{O}_{4}$ layer in the $\mathrm{Fe}_{3} \mathrm{O}_{4} / \mathrm{Pt}$ sample after considering the effect of shunting of Pt in equation 5. Adapted from Ramos et al. [47]

The amount of ANE suppression can be estimated by considering the expression of the charge current density $\left(J_{\mathrm{m}}^{\mathrm{i}}\right)$ under applied electric fields $\left(E^{\mathrm{j}}\right)$ and thermal gradients $\left(\nabla_{\mathrm{k}} T\right)$ in the linear response region: $J_{\mathrm{m}}^{\mathrm{i}}=\sigma_{\mathrm{m}}^{\mathrm{ij}} E^{\mathrm{j}}-\alpha_{\mathrm{m}}^{\mathrm{ik}} \nabla_{\mathrm{k}} T$, where $\sigma_{\mathrm{m}}^{\mathrm{jj}}$ and $\alpha^{\mathrm{ik}}$ are the elements of the electric and thermoelectric conductivity tensors, respectively. This expression can be expanded, under the conditions of the SSE in a FM/NM bilayer, to obtain the following relations:

$$
\begin{gathered}
J_{\mathrm{FM}}^{\mathrm{z}}=\sigma_{\mathrm{FM}}^{\mathrm{zz}} E^{\mathrm{z}}+\sigma_{\mathrm{FM}}^{\mathrm{zy}} E^{\mathrm{y}}-\alpha_{\mathrm{FM}}^{\mathrm{zz}}\left(\nabla_{\mathrm{z}} T\right)_{\mathrm{FM}}, \\
J_{\mathrm{FM}}^{\mathrm{y}}=\sigma_{\mathrm{FM}}^{\mathrm{yz}} E^{\mathrm{z}}+\sigma_{\mathrm{FM}}^{\mathrm{yy}} E^{\mathrm{y}}-\alpha_{\mathrm{FM}}^{\mathrm{zy}}\left(\nabla_{\mathrm{z}} T\right)_{\mathrm{FM}}, \\
J_{\mathrm{NM}}^{\mathrm{z}}=\sigma_{\mathrm{NM}}^{\mathrm{zz}} E^{\mathrm{z}}-\alpha^{\mathrm{zz}}{ }_{\mathrm{NM}}\left(\nabla_{\mathrm{z}} T\right)_{\mathrm{NM}}, \\
J_{\mathrm{NM}}^{\mathrm{y}}=\sigma_{\mathrm{NM}}^{\mathrm{yy}} E^{\mathrm{y}} .
\end{gathered}
$$

The SSE measurement is performed in open circuit condition: $I^{z}=A J_{\mathrm{FM}}^{z}=A J_{\mathrm{NM}}^{z}=0$ and $I^{y}=S_{\mathrm{FM}} J_{\mathrm{FM}}^{\mathrm{y}}+$ $S_{\mathrm{NM}} J^{\mathrm{y}}{ }_{\mathrm{NM}}=0$, where $A=L_{\mathrm{x}} L_{\mathrm{y}}$ is the area of the junction normal to the film surface and $S_{\mathrm{m}}=L_{\mathrm{x}} t_{\mathrm{m}}$ is the area along the y direction, where $t_{\mathrm{m}}(\mathrm{m}: \mathrm{FM}, \mathrm{NM})$ represents the thickness of each of the layers of the 
FM/NM bilayer. Solving equation (4) with the open circuit condition, we obtain the following expression for the electric field in the normal metal due to the ANE of the ferromagnet [86]:

$$
E^{\mathrm{y}}=r /(1+r) E_{\mathrm{ANE}}
$$

where $r=\left(\rho_{\mathrm{NM}} / \rho_{\mathrm{FM}}\right)\left(t_{\mathrm{FM}} / t_{\mathrm{NM}}\right)$ accounts for the degree of suppression of the ANE electric field from the FM layer by the NM layer (shunting factor), with $\rho_{\mathrm{FM} / \mathrm{NM}}=1 / \sigma_{\mathrm{FM} / \mathrm{NM}}$ being the electrical resistivity of the FM/NM layer. Considering the values of resistivity and thickness for the films in the $\mathrm{Fe}_{3} \mathrm{O}_{4} / \mathrm{Pt}$ bilayers, we can estimate the voltage contribution from the ANE of the $\mathrm{Fe}_{3} \mathrm{O}_{4}$ film, accounting for about $0.4 \%$ of the SSE voltage measured in the $\mathrm{Fe}_{3} \mathrm{O}_{4} / \mathrm{Pt}$ bilayer (see figure 5(a)), clearly showing that the transversal thermoelectric voltage in this system is mainly driven by the SSE effect. Moreover, we measured the ANE in the perpendicular magnetized (PM) configuration, performed by interchanging the directions of $\nabla T(\| \mathrm{x})$ and $\mathbf{H}(\| \mathrm{z})$ (see inset of figure 5(b)). In this configuration only the ANE is present, since the SSE cannot be detected due to the ISHE geometry: $\mathbf{s}\left\|\mathbf{J}_{\mathrm{S}}\right\| \mathrm{z}$ due to $\mathbf{M} \| \mathrm{z}$, see equation 1. The result shows that the ANE in the $\mathrm{Fe}_{3} \mathrm{O}_{4} / \mathrm{Pt}$ bilayer is negligible (see figure 5(b)), this is in agreement with a dominant SSE thermoelectric response in $\mathrm{Fe}_{3} \mathrm{O}_{4} / \mathrm{Pt}$ junction systems.

\subsubsection{Multilayers}

We have previously studied multilayers formed by repeated growth of $\left[\mathrm{Fe}_{3} \mathrm{O}_{4} / \mathrm{Pt}\right]$ bilayer structures as schematically depicted in figure 6(a). We have observed that in the multilayers is possible to obtain both an enhancement of the SSE voltage (see figure 6(b)) and reduction of device resistance [47], therefore achieving improved thermoelectric power output characteristics in this type of systems [48]. The reduction of the multilayer resistance is expected, since the system can be understood as a set of thin film layers electrically connected in parallel. However, the observed voltage enhancement is quite surprising and cannot be explained by a simple picture of thermal spin injection at the FM/NM interface. In this picture, the multilayer is treated as a set of independent $\mathrm{Fe}_{3} \mathrm{O}_{4} / \mathrm{Pt}$ bilayers connected in parallel with a constant heat flow, and therefore the magnitude of the injected spin currents at the $\mathrm{Fe}_{3} \mathrm{O}_{4} / \mathrm{Pt}$ interfaces considered to be constant (see figure 6(d)), then a voltage enhancement is only expected for the inner Pt layers due to additive spin current injection from both top and bottom $\mathrm{Fe}_{3} \mathrm{O}_{4} / \mathrm{Pt}$ interfaces. In the limiting case of a multilayer consisting of a very large number of bilayers, the maximum expected voltage in this scenario would be $V \approx 2 V_{\mathrm{BL}}$ (where $V_{\mathrm{BL}}$ refers to the SSE voltage of a single $\mathrm{Fe}_{3} \mathrm{O}_{4} / \mathrm{Pt}$ bilayer). 

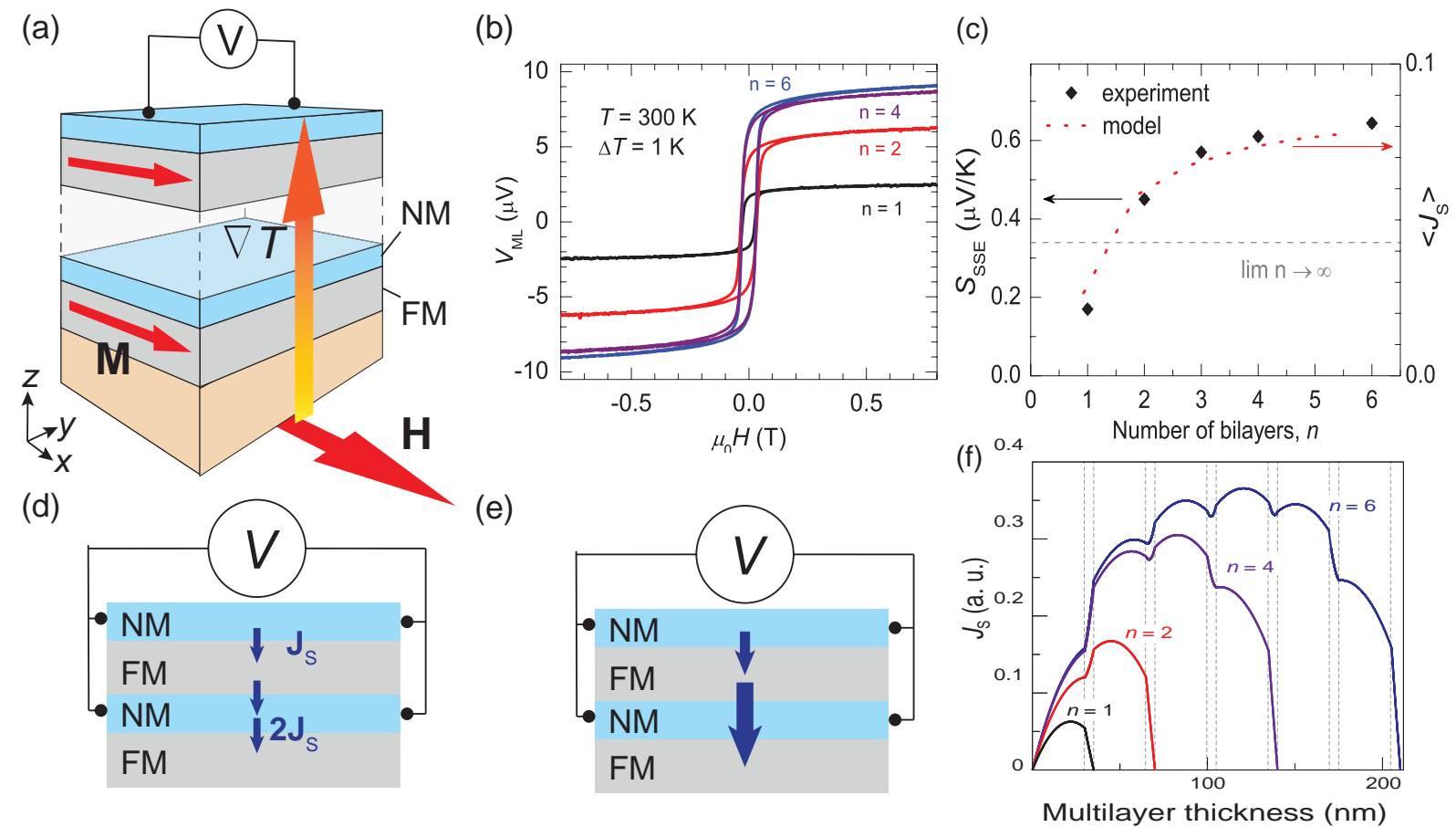

Figure 6. (a) Schematic representation of the multilayer structures for the SSE measurements. (b) Magnetic field dependence of the SSE voltage measured at $300 \mathrm{~K}$ for the $\left[\mathrm{Fe}_{3} \mathrm{O}_{4}(34) / \mathrm{Pt}(17)\right]_{\mathrm{n}}$ multilayers for different number of bilayers $\mathrm{n}$ (thickness in $\mathrm{nm})$ and $(\mathrm{c})$ the $n$ dependence of the SSE coefficient obtained from the SSE voltage value at $0.7 \mathrm{~T}\left(S_{\mathrm{SSE}}=\left(V_{\mathrm{ML}}(0.7) / \Delta T\right)\left(L_{z} / L_{y}\right)\right)$. The red dot line shows the qualitative comparison to the theoretical model of the multilayer SSE and the grey dashed line shows the expected maximum enhancement from (d) a simple thermal spin current injection picture in multilayers. (e) Schematic representation of the spin current enhancement in the FM/NM multilayer system (size of arrow proportional to spin current magnitude and (f) spin current profile across the multilayer from our theoretical model. Adapted from Ramos et al. [47]

Figure 6(b) shows the magnetic field dependence of the SSE voltage of a set of $\left[\mathrm{Fe}_{3} \mathrm{O}_{4} / \mathrm{Pt}\right]_{\mathrm{n}}$ multilayers measured at room temperature, it can be observed that the voltage increases significantly upon increasing the number of bilayers $n$, with more than a $4 \times$ increase between a single bilayer $(n=1)$ and a multilayer with $n=6$. This is quite unexpected and clearly exceeds the maximum expected value $(V \approx$ $2 V_{\mathrm{BL}}$, for $\mathrm{n} \rightarrow \infty$ ) considering the simple picture described above. Figure 6(c) shows the measured SSE coefficient (defined as $\left.S_{\mathrm{SSE}}=\left(V_{\mathrm{ML}} / \Delta \mathrm{T}\right) /\left(L_{\mathrm{z}} / L_{\mathrm{y}}\right)\right)$ obtained from the saturating field value for the different multilayers, we can see that even for $\mathrm{n}=2$ the measured voltage clearly exceeds $2 V_{\mathrm{BL}}$. Similar voltage enhancements have been also reported in other multilayer systems based on all-metal $[94,95]$ and alloxide layers [97], however in these systems the contributions from ANE and SSE cannot be easily separated, due to the similar resistivities of the FM and NM layers.

Now, let us discuss the different possibilities behind the observed SSE enhancement in the multilayers. One possibility could be a decrease of the thermal conductivity in the multilayers due to the increased number of interfaces, however thermal transport measurements in the $\left[\mathrm{Fe}_{3} \mathrm{O}_{4} / \mathrm{Pt}\right]_{\mathrm{n}}$ multilayers have shown an almost independent value of the thermal conductivity with the number of $\mathrm{Fe}_{3} \mathrm{O}_{4} / \mathrm{Pt}$ interfaces [47], suggesting that other mechanism must be responsible for the observed SSE voltage enhancement. Another possibility is the modulation of the spin transport across the multilayer thickness. To further explore this possibility, the effect of a spin current suppression in the multilayers was studied. This was performed by replacing the inner Pt interlayers of the sample for thick $\mathrm{MgO}$ layers, leaving only the topmost Pt layer, therefore heat transport across the multilayer thickness was maintained $(\mathrm{MgO}$ is a good thermal conductor), while the electron and spin transport across the multilayer thickness is suppressed. The results of this measurement are shown in figure 7, where the measured SSE voltages 
of a $\mathrm{Fe}_{3} \mathrm{O}_{4} / \mathrm{Pt}$ bilayer, a $\left[\mathrm{Fe}_{3} \mathrm{O}_{4} / \mathrm{Pt}\right]_{3}$ multilayer and a $\left[\mathrm{Fe}_{3} \mathrm{O}_{4} / \mathrm{MgO}\right]_{2} / \mathrm{Fe}_{3} \mathrm{O}_{4} / \mathrm{Pt}$ multilayer are compared. The SSE voltage shows a strong suppression upon insertion of $\mathrm{MgO}$ interlayers, as a consequence of the absence of spin current propagation through the $\mathrm{MgO}$ interlayers, the obtained SSE voltage is comparable to that of a single $\mathrm{Fe}_{3} \mathrm{O}_{4} / \mathrm{Pt}$ bilayer. This result points to the spin transport across the multilayer thickness as the possible origin for the observed SSE enhancement, in which the existence of multiple $\mathrm{Fe}_{3} \mathrm{O}_{4} / \mathrm{Pt}$ interfaces is a relevant factor.

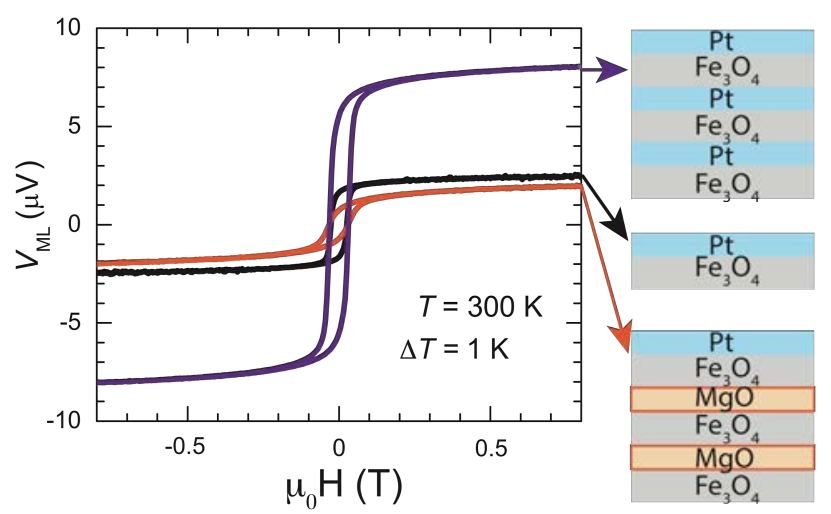

Figure 7. Effect of insertion of an electrically insulating $8 \mathrm{~nm}$ thick $\mathrm{MgO}$ layer between the $\mathrm{Fe}_{3} \mathrm{O}_{4}$ layers, only the topmost $\mathrm{Pt}$ layer is kept for the detection of the SSE on the multilayer SSE. The samples compared are a $\mathrm{Fe}_{3} \mathrm{O}_{4} / \mathrm{Pt}$ bilayer, a $\left[\mathrm{Fe}_{3} \mathrm{O}_{4} / \mathrm{Pt}_{3}\right.$ and $\mathrm{a}\left[\mathrm{Fe}_{3} \mathrm{O}_{4} / \mathrm{MgO}_{2} / \mathrm{Fe}_{3} \mathrm{O}_{4} / \mathrm{Pt}\right.$ multilayers. The layer thicknesses are 34 and $17 \mathrm{~nm}$ for $\mathrm{Fe}_{3} \mathrm{O}_{4}$ and $\mathrm{Pt}$, respectively. Adapted from Ramos et al. [47]

Now let us briefly introduce the details of the model used to qualitatively explain the physics behind the SSE voltage enhancement in $[\mathrm{FM} / \mathrm{NM}]_{\mathrm{n}}$ multilayers. In this model, two types of spin currents are considered: magnon spin currents $\left(J_{\mathrm{M}}\right)$ in the FM layers $[8,43,44,45,46]$ and conduction-electron spin currents in the NM layers $\left(J_{\mathrm{S}}\right)$. Then, we consider boundary conditions for the spin current propagating across the FM/NM interfaces: continuity of the magnon and conduction-electron spin currents at the different FM/NM (NM/FM) interfaces of the multilayer [43, 44, 45, 46, 98] and the cancellation of the magnon and conduction-electron spin currents at the bottom and top surfaces of the multilayer structure. The above conditions result in a modification of the magnitude and spatial profile of the spin current: the continuous and non-vanishing spin current at the inner FM/NM interfaces of the multilayer results in an enhancement of the spin current, with maximum values at the inner layers of the multilayer (see the supplementary information of Ref. [47] for a detailed discussion). A schematic of the predicted spin current profile using our model is shown in figure 6(f), the effect can be understood by the appearance of a new length scale that characterizes the overall variation of the spin current in the multilayer, and the presence of NM layers within the multilayer that electrically detect the large spin currents at the inner layers of the multilayer. The effectively measured SSE is an average over all the NM layers, due to the parallel contact between all the layers, and thus proportional to the average spin current over all the NM layers $\left(\left\langle J_{\mathrm{S}}\right\rangle\right)$. The predicted values of the average spin current are shown in figure 6(c), we can clearly see that the model qualitatively reproduces the observed dependence of the SSE on the number of bilayers $n$.

\section{Spin Peltier effect in Fe-based binary spinel oxides: $\mathrm{Fe}_{3} \mathrm{O}_{4} / \mathrm{Pt}$ system}

As described previously, the SPE refers to the generation of a heat current as a result of a spin current injected across the NM/FM interface. The spin current is normally generated by means of the spin Hall 
effect in the NM layer [33, 34], which induces a spin accumulation near the NM/FM interface and thus injects a spin current into the FM layer.

In this section, we will discuss the results of the spin Peltier measurements in the $\left[\mathrm{Fe}_{3} \mathrm{O}_{4} / \mathrm{Pt}\right]_{\mathrm{n}}$ systems using the lock-in thermography (LIT) technique, reported in [23]. Let us first describe the phenomenological behavior of the SPE in the LIT measurements with focusing on the results obtained for the $\mathrm{n}=6$ multilayer system. Figures $8(\mathrm{a})$ and $8(\mathrm{~b})$ show the amplitude $(A)$ and phase $(\phi)$ images for a magnetic field of $\mu_{0} H=1 \mathrm{~T}$ applied along the $+x$ direction, we can see a clear temperature modulation appearing with a phase of $\phi=0^{\circ}$. Interestingly, upon reversal of the applied magnetic field direction ( $\mathbf{H}$ $\|-x$ ), the phase changes by $180^{\circ}$, while the magnitude of $A$ remains constant (figures $8(\mathrm{c})$ and $8(\mathrm{~d})$ ). Figures 8(e) and 8(f) show the comparison of the magnetic field dependence of $A$ and $\phi$ to the magnetization of the $\mathrm{n}=6$ sample, respectively. It can be clearly seen that the magnitude of $A$ traces the magnetization of the sample and that the sign of the current-induced temperature modulation on the sample surface is reversed $\left(180^{\circ}\right.$ phase shift) upon reversal of the magnetization of the sample. This result is consistent with the expected behavior of the SPE, thus confirming the spin origin of the measured temperature modulation, further details can be found in Ref. [23].

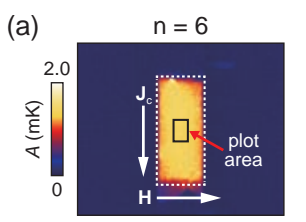

(b)

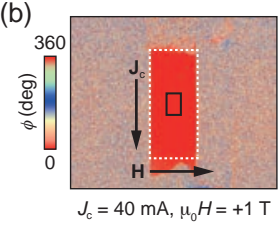

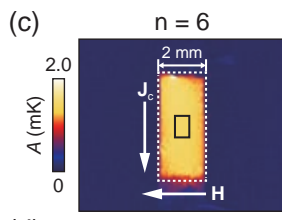

(d)

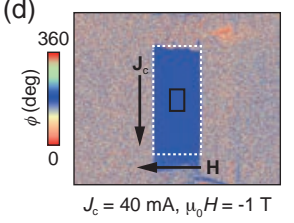

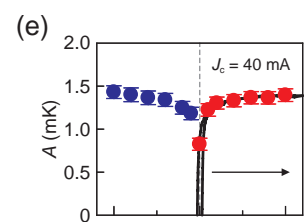

(f)

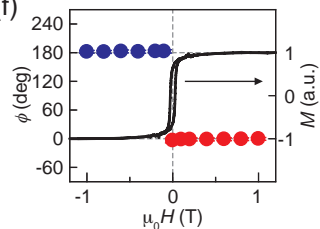

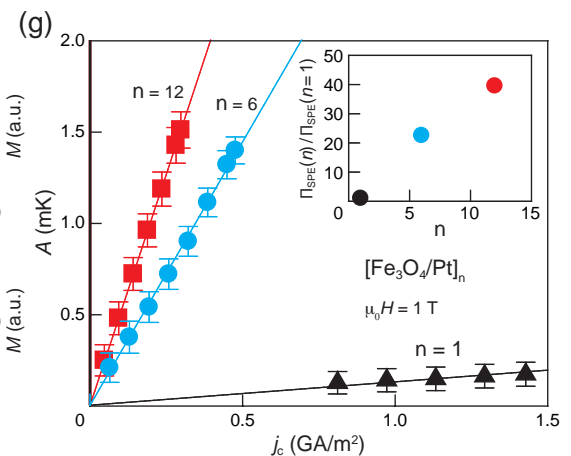

Figure 8. (a) and (b) Amplitude $A$ and phase $f$ images of the lock-in thermography measurements for the $\left[\mathrm{Fe}_{3} \mathrm{O}_{4}(23) / \mathrm{Pt}(7)\right]_{6}$ sample (thickness in $\mathrm{nm}$ ). (c) and (d) show the $A$ and $f$ measured upon reversal of the magnetic field direction. (e) and (f) Comparison of the magnetic field dependence of $A$ and $f$ to the magnetization of the sample. $(\mathrm{g})$ Dependence of $A$ on the applied charge current density for $n=1,6$ and 12 . The inset shows the $n$ dependence of the SPE enhancement $\left(\Pi_{\text {SPE }}(n) / \Pi_{\text {SPE }}(1)\right)$, where $\Pi_{\text {SPE }}(n)$ is given by the slope of the curves for each $n$. Adapted from Uchida et al. [23].

Now let us move to the dependence of the SPE magnitude on the number of bilayers $n$. We can clearly observe a dramatic increase in the magnitude of $A$ with an increasing number of bilayers $\mathrm{n}$. In figure $8(\mathrm{~g})$ the dependence of the SPE with the magnitude of the applied electric current density $\left(j_{\mathrm{c}}\right)$ is also shown for three different samples $(n=1,6$ and 12), showing a linear dependence (consistent with the characteristics of the SPE). The slope of these curves gives the SPE magnitude per charge current density $\left(\Pi_{\mathrm{SPE}}\right)$ as a function of number of bilayers $\mathrm{n}$. This is shown in the inset of figure $9(\mathrm{~g})$ after normalizing vs the value for $\mathrm{n}=1$, which gives the enhancement factor of the $\operatorname{SPE}\left(\Pi_{\mathrm{SPE}}(\mathrm{n}) / \Pi_{\mathrm{SPE}}(1)\right)$. A strong increase, reaching a factor of 40 for $\mathrm{n}=12$, is observed. The observed SPE enhancement cannot be explained by considering the multilayers as stacks of independent $\mathrm{Fe}_{3} \mathrm{O}_{4} / \mathrm{Pt}$ bilayers, which gives an enhancement ratio of only 23 (proportional to the number of interfaces $2 \mathrm{n}-1$ ). The observed unconventional enhancement can be explained using a mechanism similar to that used for the multilayer SSE [47], consisting on a redistribution of the magnon and electron spin currents across the multilayer thickness as described in the previous section. These results demonstrate the reciprocity of the SSE and SPE in multilayer systems and pave the way for realization of spin-current-based thermoelectric conversion devices. 


\section{Towards improved SSE thermoelectric conversion:}

\subsection{Common approaches}

The SSE has potential advantages over conventional thermoelectric conversion technologies for heat energy harvesting or sensing applications due to its observation in magnetic insulators [4]. Moreover, the experimental geometry, with independent and perpendicular paths for the heat and electric currents, allows to have two materials that can be optimized independently and easy implementation of thin film and flexible devices over large surfaces by thin film coating $[99,100]$. However, one obstacle for the implementation of thermoelectric conversion devices based on the SSE is the low magnitude of the SSE thermopower. Several approaches are currently being explored in this direction [12]; these include increasing the spin-current detection efficiency by using metallic layers with improved spin Hall angle characteristics [101, 102, 103], spin momentum locking in topological insulators [104, 105], hybrid ANE/SSE thermoelectric generation in thin films or bulk composite systems [96, 106, 107, 108], spin Hall thermopiles (figure 10(a)) [40, 109], and [FM/NM $]_{n}$ multilayers [23, 47, 48, 49, 94, 95, 97].

The spin Hall thermopiles improve the electrical detection of the SSE by enhancing the SSE voltage. This is achieved by using two different materials with different spin Hall angles, that are alternatively placed on top of a ferromagnetic material connected in series forming a zig-zag structure (see figure 9(a)) $[48,109]$. Figure 9(b) shows the comparison of the SSE thermoelectric voltage response between a single $\mathrm{Fe}_{3} \mathrm{O}_{4} / \mathrm{Pt}$ bilayer and a spin Hall thermopile comprising a set of 6 parallel $\mathrm{Fe}_{3} \mathrm{O}_{4} / \mathrm{Pt}$ wires connected in series forming a zig-zag structure using a metal with negligible spin Hall angle $(130 \mathrm{~nm}$ of Al) (see inset of figure 9(a) for an optical image). We observe a clear enhancement of the SSE voltage in the thermopile structure with thermoelectric voltages of $10 \mu \mathrm{V} / \mathrm{K}$ and $50 \mu \mathrm{V} / \mathrm{K}$ for the single bilayer and thermopile structure, respectively. In the zig-zag structure, the estimated SSE voltage per $\mathrm{Fe}_{3} \mathrm{O}_{4} / \mathrm{Pt}$ wire is $\sim 8.2 \mu \mathrm{V} / \mathrm{K}$, obtaining a value of the electric field per unit of temperature difference $V / L_{\mathrm{y}} \Delta T \sim$ $1.6 \mathrm{mV} / \mathrm{Km}$, which is consistent with the electric field of the $\mathrm{Fe}_{3} \mathrm{O}_{4} / \mathrm{Pt}$ bilayer, therefore implying that the larger SSE voltage in the thermopile is a consequence of an increased effective length of the sample and that further enhancements can be obtained by increasing the wire integration density of the devices [109]. Despite the increase of the SSE voltage in the spin Hall thermopile devices, the output power, obtained by measuring the SSE voltage on a resistive load $\left(R_{\mathrm{L}}\right)$ attached to the SSE device (inset of figure 9(c)), shows no improvement when compared to the single bilayer case (figure 9(c)). This situation arises as a consequence of the fact that, in the thermopile structures, the effective device area is reduced in the patterned device and the voltage enhancement is due to the increase in effective length of the sample, resulting in an increase of the internal resistance of the thermopile [13], which strongly limits the extractable power of these devices. 


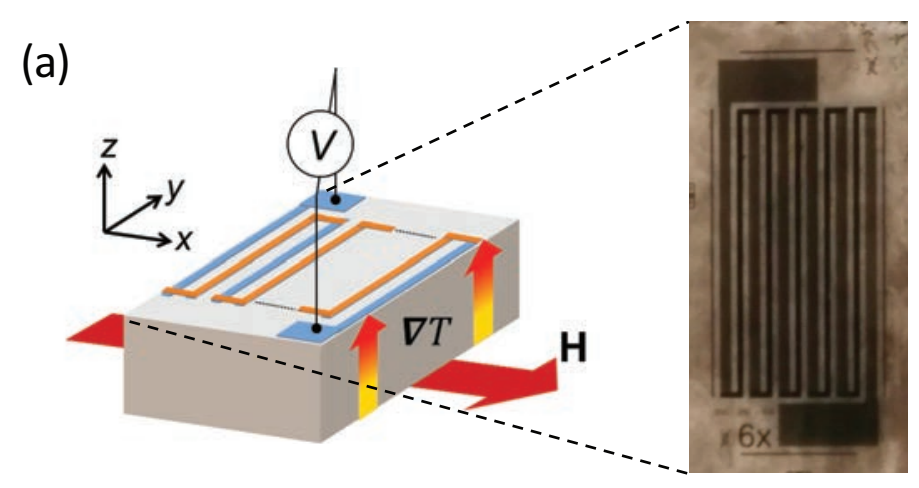

(b)

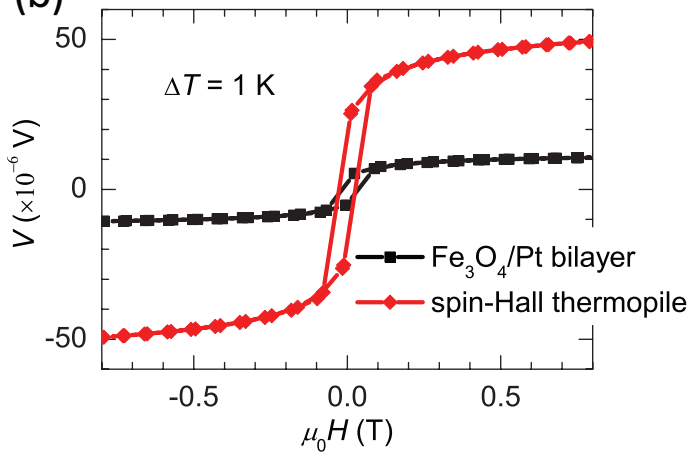

(c)

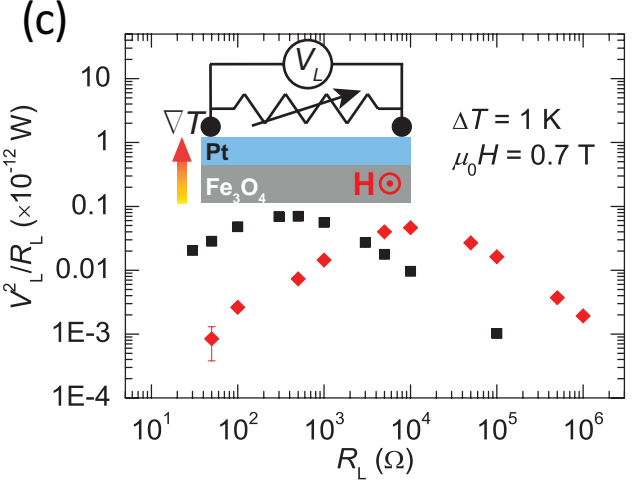

Figure 9. Illustration of the SSE measurement in a (a) spin Hall thermopile system (the optical image shows a Fe ${ }_{3} \mathrm{O}_{4} / \mathrm{Pt}$ thermopile with $\mathrm{Al}(130)$ interconnecting wires. (b) Magnetic field dependence of the SSE voltage measured at $300 \mathrm{~K}$ and with an applied temperature difference of $\Delta T=1 \mathrm{~K}$ in a $\mathrm{Fe}_{3} \mathrm{O}_{4}(50) / \mathrm{Pt}(5)$ and a spin Hall thermopile fabricated on the same bilayer (thickness in $\mathrm{nm}$ ). (c) Thermoelectric power output characteristics of the bilayer and the spin Hall thermopile structures measured as a function of the magnitude of a variable load resistance $\left(R_{\mathrm{L}}\right)$ connected to the device under test (see inset). Adapted from Ramos et al. [48]

\subsection{Our approach: $\left[\mathrm{Fe}_{3} \mathrm{O}_{4} / \mathrm{Pt}\right]_{n}$ multilayer}

We have seen that the SSE-driven thermoelectric voltage in $\left[\mathrm{Fe}_{3} \mathrm{O}_{4} / \mathrm{Pt}\right]_{\mathrm{n}}$ multilayers is strongly enhanced as a consequence of a possible spin-current increase within the multilayers. In fact, a reduction of the Pt thickness results in a larger SSE voltage (see figure 10(a)). Moreover, in the multilayer system, the voltage increase is accompanied by a reduction of the sample resistance, thus an increase of the extractable thermoelectric power from the device is expected. We estimated the deliverable thermoelectric power of the $\left[\mathrm{Fe}_{3} \mathrm{O}_{4} / \mathrm{Pt}\right]_{\mathrm{n}}$ multilayers by performing measurements of the voltage on a variable resistive load connected to the multilayers, as previously done for the thermopile structures (inset of figure 9(c)). Figure 10(b) shows that the output power characteristics of the $\left[\mathrm{Fe}_{3} \mathrm{O}_{4} / \mathrm{Pt}\right]_{\mathrm{n}}$ multilayers are largely improved, with an enhancement of more than two orders of magnitude from the single bilayer $(n=1)$ to the multilayer with $n=12$. This result demonstrates that the overall thermoelectric performance of the multilayers has clear advantages with respect to a single FM/NM bilayer and spin Hall thermopile structures.

According to the multilayer SSE model, the enhancement of the SSE in multilayers is expected to be strongly dependent on the spin transport characteristics in FM and PM layers, as we have observed in temperature dependent measurements [49]. Here, an increase of the multilayer enhancement ratio was observed to increase with decreasing the temperature, in agreement with larger characteristic lengths for spin transport in $\mathrm{Fe}_{3} \mathrm{O}_{4}$ and $\mathrm{Pt}$ at lower temperatures [93, 110]. This result suggests that there is room for further improvement of the multilayer SSE performance by selection of materials with better spin transport characteristics. 

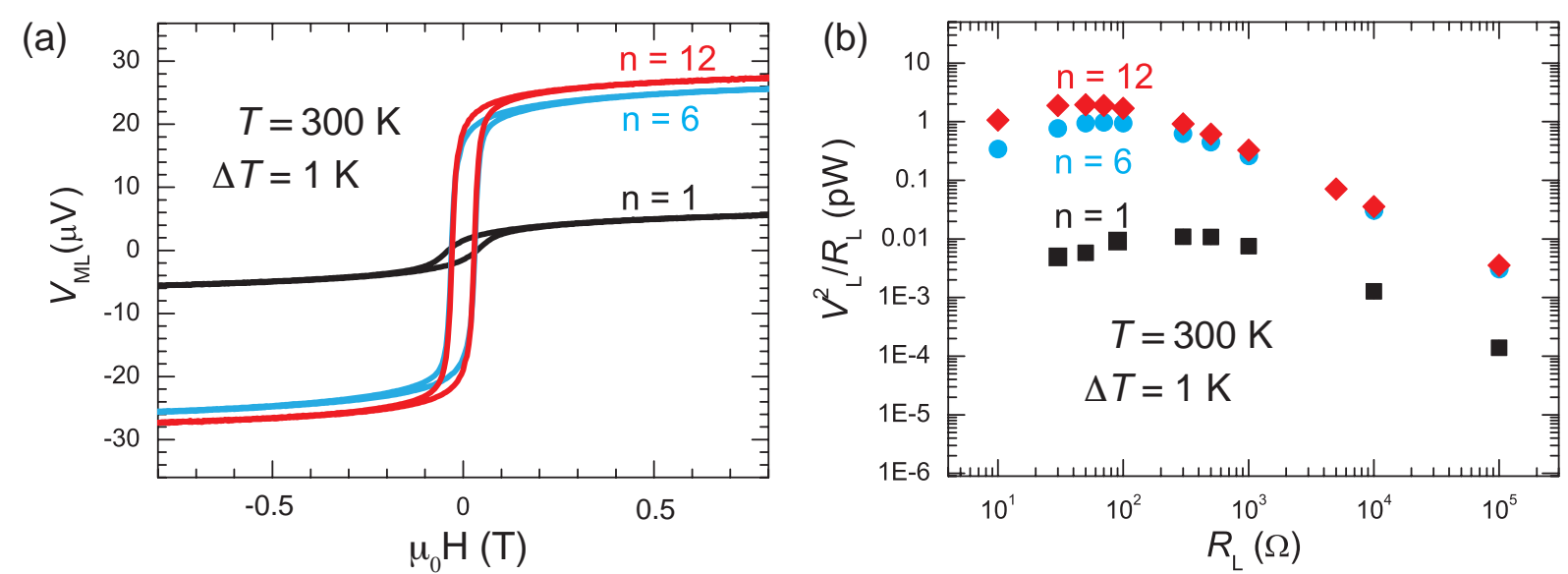

Figure 10 (a) Magnetic field dependence of the SSE voltage in $\left[\mathrm{Fe}_{3} \mathrm{O}_{4}(23) / \mathrm{Pt}(7)\right]_{n}$ multilayers with $\mathrm{n}=1,6$ and 12 (thickness in $\mathrm{nm}$ ) and their (c) thermoelectric power output characteristics determined from the voltage measured on a variable load resistance as shown in the inset of figure 10(d) (figure shows values estimated from SSE voltage at $H=0.7 \mathrm{~T}$ ). Adapted from Ramos et al. [48]

\section{Spin Seebeck effects in other magnetically-ordered materials.}

In the previous sections, we have described the SSE in FM/NM-based bilayer and multilayer systems, where FM is a ferro- or ferri-magnet material, and NM is a normal metal (NM). More recently, heat driven spin currents by the SSE have been observed in a wider range of materials presenting different types of magnetically ordered arrangements, these include: compensated ferrimagnets, antiferromagnets, paramagnets, systems with frustrated magnetic order and amorphous materials [50, $51,111,112,113,114]$. In this section, we will briefly provide an overview of these recent developments.

As previously shown in section 2.1, the SSE was initially formulated in terms of conduction electrons [11], however with the observation of the SSE in magnetic insulators the theoretical framework was reformulated in terms of temperature differences between the magnon and electron systems in FM and PM, respectively [35, 36, 37]. It is now commonly agreed that the SSE results from the thermal generation of magnon spin currents in the FM and electrically converted by the ISHE in the NM [43, $44,45,46,65,115]$. Usually the FM insulator is treated as a conventional ferromagnet with a net magnetization, despite its ferri-magnetic nature, with more than one magnetically ordered sublattice $[116,117]$.

One example of the observation of the multiple magnetic sublattice nature of a ferrimagnet by the SSE, is illustrated by measurements in $\mathrm{Gd}_{3} \mathrm{Fe}_{5} \mathrm{O}_{12}$ [111], a compensated ferrimagnetic oxide with a magnetic compensation point at a temperature of around $T_{\mathrm{c}} \sim 288 \mathrm{~K}$ [118]. In this system, the SSE shows a sign reversal as the temperature is decreased across the magnetic compensation point $\left(T_{\mathrm{c}}\right)$, demonstrating the dependence of the SSE on the individual magnetization of the sublattices of the ferrimagnet, in agreement with theoretical predictions [56]. Furthermore, as the temperature is further reduced an additional sign reversal was observed, which was explained as a consequence of the spin current induced by higher-energy magnon modes of the material, proving the dependence of the SSE on the complex spin wave dynamics in ferrimagnets [111].

The dependence of the SSE on the details of the spin wave spectrum has been further demonstrated by 
measurements of the SSE in antiferromagnetically ordered materials [50, 51]. In these materials two equivalent sublattices with opposite spin polarizations are present, giving rise to two overlapping spin wave dispersions with opposite spin polarizations, application of a relatively strong field can split these spin wave modes, where the frequency of the modes is increased/decreased depending on the relative orientation of the spin polarization with the applied magnetic field, and results in the generation of a net spin current [52]. This antiferromagnetic-spin-wave-driven SSE has been experimentally demonstrated for $\mathrm{Cr}_{2} \mathrm{O}_{3}$ [50] and $\mathrm{MnF}_{2}$ [51], these studies also report the observation of a characteristic spin-flop induced voltage when a large magnetic field is applied along the crystalline easy axis of the antiferromagnet, thus demonstrating the antiferromagnetic origin of the observed signal.

Common SSE theories rely on long-range magnetically ordered materials, however recent studies show the existence of a SSE in insulating paramagnetic oxides [112] and magnetically frustrated systems (one-dimensional spin-1/2 chains) [113], where no long-range magnetic order is present. The observed SSE is understood as a result of short ranged magnetic correlations which can drive spin currents, however the origin of the SSE in paramagnets and one-dimensional spin-1/2 chain systems is fundamentally different, being driven by a spinon spin current in the latter case [113]. In light of the above, it is also interesting to mention the recent observation of a spin-transport in an amorphous magnetic insulator [114]. The SSE was also previously observed in a non-magnetic semiconductor [119] at low temperatures and high magnetic fields, however its origin is fundamentally different from the SSE phenomena discussed here.

Another parameter that can be tuned in the SSE is the materials used for electrical detection of the thermally generated spin current by the ISHE, where Pt is the most commonly used material, as the spin current detection efficiency is relatively large in this metal $[33,34]$. Recently, the ISHE has also been investigated in antiferromagnetic $[120,121,122]$ and ferromagnetic metals $[105,106,107,123,124$, $125,126,127]$, these studies open the possibility to explore a wider range of materials which are fundamentally interesting and can also possibly lead to increased thermoelectric conversion efficiency of SSE devices, due to combined thermoelectric generation by means of the SSE and ANE.

\section{Summary and outlook.}

We have investigated thermo-spin effects in Fe-oxide based heterostructures. We have mainly focused on the results of the thermos-spin conversion in multilayers formed by repeated growth of FM/NM bilayers, particularly $\left[\mathrm{Fe}_{3} \mathrm{O}_{4} / \mathrm{Pt}\right]_{\mathrm{n}}$-based systems. Measurements of the SSE and SPE in such multilayer systems present a strong enhancement of the thermoelectric conversion response; this enhancement cannot be simply explained by the system geometry, but other factors need to be taken in consideration. Basically, the propagation of the spin current in the multilayer geometry allows for an enhancement of the spin current in the middle layers of the multilayer structure, due to the continuity and non-zero value of the spin current in the inner interfaces of the multilayer.

We have shown the results on the thermoelectric response of spin Hall thermopiles and multilayer systems: in the former case the sensitivity is increase at the expense of a device resistance increase, resulting in no improvement in the deliverable thermoelectric power. However, the multilayer system with a simultaneous increase in the thermoelectric response and reduction of device internal resistance, was shown to improve the deliverable thermoelectric power on an external resistive load, reaching two orders of magnitude increase with respect to a single bilayer structure. This represents a significant leap in the thermoelectric efficiency of the SSE and SPE. Moreover, the observed multilayer SSE 
enhancement is expected to further improve by selection of materials with better spin transport characteristics.

Finally, we also summarize recent results of the SSE in materials with types of magnetic order other than conventional ferro-/ferri- magnetic systems. These can enrich the physical understanding of the thermo-spin effects, span spin-current studies in other areas [128, 129, 130], and possibly provide novel routes of exploration to improve the efficiency of SSE-thermoelectric conversion.

\section{Acknowledgments}

We would like to thank A. Anadón, P. Jiménez-Cavero, M. H. Aguirre, T. Kikkawa, S. Daimon, R. Iguchi, T. Oyake, J. Shiomi, H. Adachi, and S. Maekawa for helpful discussions and their collaboration. This work was supported by ERATO "Spin Quantum Rectification Project” (Grant No. JPMJER1402) and PRESTO "Phase Interfaces for Highly Efficient Energy Utilization" (Grant No. JPMJPR12C1) from JST, Japan; Grant-in-Aid for Scientific Research (A) (Grant No. JP15H02012) and Grant-in-Aid for Scientific Research on Innovative Area, "Nano Spin Conversion Science" (Grant No. JP26103005) from JSPS KAKENHI, Japan, the NEC Corporation, and the Noguchi Institute. The microscopy works were conducted in the Laboratorio de Microscopías Avanzadas at INA, Universidad de Zaragoza; H2020-MSCA-RISE-2016 SPICOLOST (Grant No. 734187); the Spanish Ministry of Economy and Competitiveness (Grant No. MAT2017-82970- C2, including FEDER), Spain; and the Aragón regional government (E26), Spain. 


\section{References}

[1] Callen H B 1985 Thermodynamics and an introduction to thermostatistics (John Wiley \& Sons, Inc)

[2] He J and Tritt T M 2017 Science 3571369

[3] Bauer G E W, Saitoh E and van Wees B J 2012 Nature Mater. 11391

[4] Boona S R, Myers R C and Heremans J P 2014 Energy Environ. Sci. 7885

[5] Maekawa S, Adachi H, Uchida K, Ieda J and Saitoh E 2013 J. Phys. Soc. Japan 82102002

[6] Maekawa S, Saitoh E, Valenzuela S O and Kimura T 2012 Spin Current (Oxford, Oxford

University Press)

[7] Uchida K et al. 2010 Nature Mater. 9894

[8] Kajiwara Y et al. 2010 Nature 464262

[9] Saitoh E, Ueda M, Miyajima H and Tatara G 2006 Appl. Phys. Lett. 88182509

[10] Tserkovnyak Y, Brataas A, Bauer G E W and Halperin B I 2005 Rev. Mod. Phys. 771375

[11] Uchida K, Takahashi S, Harii K, Ieda J, Koshibae W, Ando K, Maekawa S and Saitoh E 2008 Nature 455, 778

[12] Uchida K, Ishida M, Kikkawa T, Kirihara A, Murakami T and Saitoh E 2016 Proc. IEEE 104 1946

[13] Uchida K, Ishida M, Kikkawa T, Kirihara A, Murakami T and Saitoh E 2014 J. Phys.: Condens. Matter 26343202

[14] Uchida K, Adachi H, An T, Ota T, Toda M, Hillebrands B, Maekawa S and Saitoh E 2011

Nature Mater. 10737

[15] Uchida K, Adachi H, An T, Nakayama H, Toda M, Hillebrands B, Maekawa S and Saitoh E 2012 J. Appl. Phys. 111053903

[16] Weiler M, Huebl H, Goerg F S, Czeschka F D, Gross R and Goennenwein S T B 2012 Phys. Rev. Lett. 108176601

[17] Azevedo A, Vilela Leão L H, Oliveira A B and Rezende S M 2005 J. Appl. Phys. 97 10C715

[18] Costache M V, Sladkov M, Watts S M, van der Wal C H and van Wees B J 2006 Phys. Rev. Lett. 97216603

[19] Valenzuela S O and Tinkham M 2006 Nature 442176

[20] Kimura T, Otani Y, Sato T, Takahashi S and Maekawa S 2007 Phys. Rev. Lett. 98156601

[21] Flipse J, Dejene F K, Wagenaar D, Bauer G E W, Ben Youssef J and van Wees B J 2016 Phys. Rev. Lett. 113027601

[22] Daimon S, Iguchi R, Hioki T, Saitoh E and Uchida K 2016 Nat. Commun. 7, 13754

[23] Uchida K et al. 2017 Phys. Rev. B 95184437

[24] Daimon S, Uchida K, Iguchi R, Hioki T and Saitoh E 2017 Phys. Rev. B 96024424

[25] Itoh R, Iguchi R, Daimon S, Oyanagi K, Uchida K and Saitoh E 2017 Phys. Rev. B 96184422

[26] Grau-Crespo R, Al-Baitai A Y, Saadoune I and De Leeuw N H 2010 J. Phys. Condens. Matter 22255401

[27] Dronskowski R 2001 Adv. Funct. Mater. 1127

[28] Walz F 2002 J. Phys.: Condens. Matter 14 R285

[29] Jin X S, Ramos R, Zhou Y, McEvoy C and Shvets I V 20069908 C509

[30] Ramos R, Arora S K and Shvets I V 2008 Phys. Rev. B 78214402

[31]Fernández-Pacheco A, De Teresa J M, Orna J, Morellón L, Algarabel P A, Pardo J A and Ibarra M R 2008 Phys. Rev. B 77, 100403

[32] Wu H C, Ramos R, Sofin R G S, Liao Z M, Abid M and Shvets I V 2012 Appl. Phys. Lett. 101 052402

[33] Hoffmann A 2013 IEEE Trans. Magn. 495172

[34] Sinova J, Valenzuela S O, Wunderlich J, Back C H and Jungwirth T 2015 Rev. Mod. Phys. 87 1213

[35] Xiao J, Bauer G E W, Uchida K, Saioth E and Maekawa S 2010 Phys. Rev. B 81214418

[36] Adachi H, Ohe J, Takahashi S and Maekawa S 2011 Phys. Rev. B 83094410

[37] Adachi H, Uchida K, Saitoh E and Maekawa S 2013 Rep. Prog. Phys. 7636501

[38] Kehlberger et al. 2015 Phys. Rev. Lett. 115096602

[39] Kikkawa T, Uchida K, Daimon S, Qiu Z, Shiomi Y and Saitoh E 2015 Phys. Rev. B 92064413 
[40] Kikkawa T et al. 2013 Phys. Rev. B 88214403

[41] Jin H, Boona S R, Yang Z, Myers R C and Heremans J P 2015 Phys. Rev. B 92054436

[42] Ritzmann U, Hinzke D, Kehlberger A, Guo E-J, Kláui M and Nowak U 2015 Phys. Rev. B 92 174411

[43] Rezende S M, Rodríguez-Suárez R L, Cunha R O, Rodrigues A R, Machado F L A, Fonseca Guerra G A, Lopez Ortiz J C and Azevedo A 2014 Phys. Rev. B 89014416

[44] Rezende S M, Rodríguez-Suárez R L, Cunha R O, Lopez Ortiz J C and Azevedo 2016 J. Magn. Magn. Mater. 400171

[45] Zhang S S-L, and Zhang S 2012 Phys. Rev. Lett. 109, 096603

[46] Zhang S S-L, and Zhang S 2012 Phys. Rev. B 86, 214424

[47] Ramos R et al. 2015 Phys. Rev. B 92 220407(R)

[48] Ramos R, Anadón A, Lucas I, Uchida K, Algarabel P A, Morellón L, Aguirre M H, Saitoh and Ibarra M R 2016 APL Mater. 4104802

[49] Ramos R et al. 2017 AIP Advances 7055915

[50] Seki S, Ideue T, Kubota M, Kozuka Y, Takagi R, Nakamura M, Kaneko Y, Kawasaki M and Tokura Y 2015 Phys. Rev. Lett. 115266601

[51] Wu S M, Zhang W, KC A, Borisov P, Pearson J E, Jiang J S, Lederman D, Hoffmann A and Bhattacharya A 2016 Phys. Rev. Lett 116097204

[52] Rezende S M and Rodríguez-Suárez R L 2015 Phys. Rev. B 93014425

[53] Rezende S M and Rodríguez-Suárez R L 2016 Phys. Rev. B 93054412

[54] Ohe J, Adachi H, Takahashi S and Maekawa S 2011 Phys. Rev. B 83115118

[55] Bender S A, Duine R A and Tserkovnyak 2012 Phys. Rev. Lett. 108246601

[56] Ohnuma Y, Adachi H, Saitoh E and Maekawa S 2013 Phys. Rev. B 87014423

[57] Chotorlishvili L, Toklikishvili Z, Dugaev V K, Barnaś J, Trimper S and Berakdar J 2013 Phys.

Rev. B 88144429

[58] Ren J 2013 Phys. Rev. B 88220406

[59] Bender S A and Tserkovnyak 2015 Phys. Rev. B 91140402

[60] Lyapilin I I, Okorokov M S and Ustinov V V 2015 Phys. Rev. B 91195309

[61] Etesami S R, Chotorlishvili L and Berakdar J 2015 Appl. Phys. Lett. 107132402

[62] Brataas A, Skarsvåg H, Tveten E G and Fjaerbu E L 2015 Phys. Rev. B 92180414

[63] Chotorlishvili L, Toklikishvili Z, Etesami S R, Dugaev V K, Barnaś J and Berakdar J 2015 J.

Magn. Magn. Mater. 396254

[64] Tserkovnyak Y, Bender S A, Duine R A and Flebus B 2016 Phys. Rev. B 93 100402(R)

[65] Cornelissen L J, Peters K J H, Bauer G E W, Duine R A and van Wees BJ 2016 Phys. Rev. B 94

014412

[66] Scharf B, Matos-Abiague A, Žutic I and Fabian J 2012 Phys. Rev. B 85085208

[67] Adachi H, Uchida K, Saitoh E, Ohe J, Takahashi S and Maekawa S 2010 Appl. Phys. Lett. 97 252506

[68] Tikhonov K S, Sinova J and Finkel'stein A M 2013 Nat. Commun. 41945

[69] Bosu S, Sakuraba Y, Uchida K, Saito K, Ota T, Saitoh E and Takanashi K 2011 Phys. Rev. B 83 224401

[70] Jaworski C M, Yang J, Mack S, Awschalom D D, Heremans J P and Myers R C 2010 Nature Mater. 9, 898

[71] Arboleda J D, Olmos O A, Aguirre M H, Ramos R, Anadón A and Ibarra M R 2016 Appl. Phys. Lett. 108232401

[72] Meier D et al. 2015 Nature Commun. 68211

[73] Bosu S, Sakuraba Y, Uchida K, Saito K, Kobayashi W, Saitoh E and Takanashi K 2012 J. Appl. Phys. 111 07B 106

[74] Wang S H, Zou L K, Cai J W, Shen B G and Sun J R 2013 Phys. Rev. B 88214304

[75] Schmid M, Srichandan S, Meier D, Kuschel T, Schmalhorst J-M, Vogel M, Reiss G, Strunk C and Back C H 2013 Phys. Rev. Lett. 111187201

[76] Giles B L, Yang Z, Jamison J S and Myers R C 2015 Phys. Rev. B 92224415

[77] Ganzhorn K et al. 2017 AIP Advances 7085102 
[78] Cornelissen L J, Oyanagi K, Kikkawa T, Qiu Z, Kuschel T, Bauer G E W, van Wees B J and Saitoh E 2017 Phys. Rev. B 96104441

[79] Weiler M et al. 2012 Phys. Rev. Lett. 108106602

[80] Lee W-L, Watauchi S, Miller V L, Cava R J and Ong N P 2004 Phys. Rev. Lett. 93226601

[81] Miyasato T, Abe N, Fujii T, Asamitsu A, Onoda S, Onose Y, Nagaosa N and Tokura Y 2007

Phys. Rev. Lett. 99086602

[82] Pu Y, Chiba D, Matsukura F, Ohno H and Shi J 2008 Phys. Rev. Lett. 101117208

[83] Mizuguchi M, Ohata S, Uchida K, Saitoh E and Takanashi K 2012 Appl Phys. Express 5093002

[84] Ramos R et al. 2014 Phys. Rev. B 90054422

[85] Fang C, Wan C H, Yuan Z H, Huang L, Zhang X, Wu H, Zhang Q T and Hou X F 2016 Phys.

Rev. B 93054420

[86] Ramos R et al. 2013 Appl. Phys. Lett. 102072413

[87] Basso V, Ferraro E, Magni A, Sola A, Kuerpferling M and Pasquale M 2016 Phys. Rev. B 93 184421

[88] Basso V, Ferraro E and Piazzi M 2016 Phys. Rev. B 94144422

[89] Ohnuma T, Matsuo M and Maekawa S 2017 Phys. Rev. B 96134412

[90] Breitenstein O, Warta W and Langenkamp M 2010 Lock-in Thermography: Basics and Use for Evaluating Electronic Devices and Materials (Berlin: Springer)

[91] Wid O, Bauer J, Muller A, Breitenstein, Parkin S S P and Schmid G 2016 Sci. Rep. 628233

[92] Jiménez-Cavero P et al. 2017 APL Mater. 5026103

[93] Anadón A, Ramos R, Lucas I, Algarabel P A, Morellón L, Ibarra M R and Aguirre M H 2016 Appl. Phys. Lett. 109012404

[94] Uchida K, Kikkawa T, Seki T, Oyake T, Shiomi J, Qiu Z, Takanashi K and Saitoh E 2015 Phys.

Rev. B 92094414

[95] Lee K-D et al. 2015 Sci. Rep. 510249

[96] Boona S R, Vandaele K, Boona I N, McComb D W and Heremans J P 2016 Nat. Commun. 7

13714

[97] Shiomi Y, Handa Y, Kikkawa T and Saitoh E 2015 Appl. Phys. Lett. 106232403

[98] Takahashi S, Saitoh E and Maekawa S 2010 J. Phys.: Conf. Ser. 200062030

[99] Kirihara A, Uchida K, Kajiwara Y, Ishida M, Nakamura Y, Manako T, Saitoh E and Yorozu S 2012 Nat. Mater. 11686

[100] Kirihara A et al. 2016 Sci. Rep. 623114

[101] Liu L, Pai C-F, Li Y, Tseng H W, Ralph D C and Buhrman R A 2012 Science 336555

[102] Niimi Y, Kawanishi Y, Wei D H, Deranlot C, Yang H X, Chshiev M, Valet T, Fert A and Otani Y 2012 Phys. Rev. Lett. 109156602

[103] Laczkowski P et al. 2014 Appl. Phys. Lett. 104142403

[104] Jiang Z, Chang C-Z, Masir M R, Tang C, Xu Y, Moodera J S, MacDonald A H and Shi J 2016

Nat. Commun. 711458

[105] Okuma N, Masir M R and MacDonald A H 2017 Phys. Rev. B 95165418

[106] Kikkawa T, Uchida K, Daimon S, Shiomi Y, Adachi H, Qiu Z, Hou D, Jin X-F, Maekawa S and Saitoh E 2013 Phys. Rev. B 88214403

[107] Miao B F, Huang S Y, Qu D and Chien C L 2013 Phys. Rev. Lett. 111066602

[108] Tian D, Li Y, Qu D, Jin X and Chien C L 2015 Appl. Phys. Lett. 106212407

[109] Uchida K, Nonaka T, Yoshino T, Kikkawa T, Kikuchi D and Saitoh E 2012 Appl. Phys.

Express 5093001

[110] Sagasta E, Omori Y, Isasa M, Gradhand M, Hueso L E, Niimi Y, Otani Y and Casanova F 2016

Phys. Rev. B 94060412

[111] Geprägs S et al. 2016 Nat. Commun. 710452

[112] Wu S M, Pearson J E and Bhattacharya 2015 Phys. Rev. Lett. 114186602

[113] Hirobe D, Sato M, Kawamata T, Siomi Y, Uchida K, Iguchi R, Koike Y, Maekawa S and

Saitoh E 2017 Nature Phys. 1330

[114] Wesenberg D, Liu T, Balzar D, Wu M and Zink B L 2017 Nature Phys. 13987

[115] Kikkawa T, Shen K, Flebus B, Duine R A, Uchida K, Qiu Z, Bauer G E W and Saitoh E 2016

Phys. Rev. Lett. 117207203 
[116] Barker J and Bauer G E W 2016 Phys. Rev. Lett. 117217201

[117] Princep A J, Ewings R A, Ward S, Tóth S, Dubs C, Prabhakaran D and Boothroyd A T 2017 npj Quantum Materials 263

[118] Pauthenet R. 1958 J. Appl. Phys. 29253

[119] Jaworski C M, Myers R C, Johnston-Halperin and Heremans J P 2012 Nature 487210

[120] Frangou L, Oyarzún S, Auffret S, Vila L, Gambarelli S and Baltz V 2016 Phys. Rev. Lett. 116 077203

[121] Zhang W, Jungfleisch M B, Jiang W, Pearson J E and Hoffmann A 2014 Phys. Rev. Lett. 113 196602

[122] Zu D, Huang S Y and Chien C L 2015 Phys. Rev. B 92020418

[123] Azevedo A, Alves Santos O, Fonseca Guerra G A, Cunha R O, Rodríguez-Suárez R and Rezende S M 2014 Appl. Phys. Lett. 104052402

[124] Wu S M, Hoffmann J, Pearson J E and Bhattacharya A 2014 Appl. Phys. Lett. 105092409

[125] Du C, Wang H, Yang F and Hammel P C 2014 Phys. Rev. B 90140407

[126] Seki T, Uchida K, Kikkawa T, Qiu Z and Saitoh E 2015 Appl. Phys. Lett. 107092401

[127] Ramos R, Wongjom P, Iguchi R, Yagmur A, Qiu Z, Pinitsoontorn S, Uchida K and Saitoh E 2018 J. Magn. Magn. Mater. 447134

[128] Walowski J and Münzenberg M 2016 J. Appl. Phys. 120140901

[129] Seifert T et al. 2017 Spin 71740010

[130] Seifert T et al. 2017 arXiv:1709.00768 [cond-mat.mes-hall] 